\title{
Semblanza de Miguel E. Schulz (1851-1922): Eminente profesor y educador
}

Elsie Rockwell

Centro de Investigación y de Estudios Avanzados

rockwell@cinvestav.mx

María Elena Maruri

Centro de Investigación y de Estudios Avanzados

emaruri@cinvestav.mx

Miguel E. Schulz nació el 11 de octubre de 1851 y falleció en la Ciudad de México a la edad de 71 años, el 3 de diciembre de 1922. ${ }^{1}$ Estudió sus primeras letras con el maestro don Joaquín Noreña; ingresó a la Academia de San Carlos y posteriormente a la Escuela Nacional Preparatoria (ENP). Desde muy joven destacó por su talento en las artes, principalmente en la escultura, y en 1870 obtuvo una pensión en la Escuela de Bellas Artes, como estímulo a su aprovechamiento. ${ }^{2}$ En 1910, después de una destacada carrera como profesor y autor, la Universidad Nacional lo honró con el grado de doctor ex officio (Garciadiego, 1996: 115-116).

Aunque su primera vocación fue estudiar arquitectura y escultura, ${ }^{3}$ Schulz se distinguió por su amplia experiencia como profesor y funcionario en el sector educativo. Inició su trayectoria en la docencia siendo profesor en la primaria privada Institución Katthain, en la que ocupó la subdirección y donde enseñó varias materias. ${ }^{4}$ A partir de 1882, y durante cuarenta años, Miguel Schulz impartió la materia de Cosmografía y Geografía en la Escuela Nacional Preparatoria ${ }^{5}$, contando con alumnos sobresalientes entre los que se mencionan a Ezequiel

\footnotetext{
1 "Obituario de Miguel E. Schulz", El Universal, 4 de diciembre de 1922. En varios documentos y textos académicos el nombre aparece como Schultz; en esta semblanza respetamos la ortografía Schulz, que aparece en la firma del documento impreso incluido en esta publicación y en su expediente.

${ }^{2}$ Archivo General de la Nación (en adelante ACN), Instrucción Pública y Bellas Artes, c. 316, exp. 8, fs. 1-1v. 1870.

${ }^{3}$ Archivo de Concentración Histórico de la Secretaría de Educación Pública (en adelante ACHSEP), Secretaría de Estado y del Despacho de Justicia e Instrucción Pública, Expediente personal (Miguel E. Schulz), exp. S2/12, f. 118. 1887-1917.

4 "Obituario..." El Universal, 4 de diciembre de 1922.

${ }^{5}$ El primer grupo estaba a cargo del ingeniero Antonio García Cubas, considerado el mejor geógrafo mexicano de su tiempo. ACHSEP, Secretaría de Estado y del Despacho de Justicia e Instrucción Pública, Expediente personal (Miguel E. Schulz), exp. S2/12, fs. 2-4. 1882
} 
A. Chávez y Gregorio Torres Quintero (Meneses, 1998: 249, 575, 767). Participó como profesor fundador de la Escuela Normal de Profesores de Instrucción Primaria en 1887, a cargo del curso de Cosmografía y Geografía General (Garciadiego, 1996: 115; Bazant, 1993: 132, 139). En 1902, el entonces director de la Escuela Nacional Preparatoria, Enrique C. Rebsamen, lo nombró profesor de Meteorología, Geografía General y Climatología y a partir de 1906 impartió, en la misma institución, los cursos de Geografía Americana y Patria, así como de Historia General. ${ }^{6}$ Además, impartió cursos en las escuelas de Agricultura y Veterinaria y ocasionalmente en el antiguo Colegio Militar y a varios grupos de la Legión de Honor.? Para 1910, Schulz era considerado el profesor más prestigiado de geografía en la Preparatoria y obtuvo la titularidad de la cátedra en $1913 .^{8}$ Ese mismo año, fue nombrado catedrático de Historia y Geografía Histórica en la Escuela de Altos Estudios de la Universidad. ${ }^{9}$

Schulz participó también en los congresos pedagógicos nacionales de la época porfiriana, pues fue delegado por Chiapas en el Segundo Congreso Nacional de Instrucción (1890-1891), así como miembro de algunas comisiones de estudio (Meneses 1998: 473-474). En dicho congreso trabajó en la reorganización de la instrucción primaria, el plan de estudios de la Escuela Normal y los estudios de preparatoria. ${ }^{10}$

Entre las publicaciones de Schulz destacan Apuntes para el Curso de Geografía (1896) 11 y el Curso general de geografía (1895), texto que durante años fue obligatorio en la materia de Geografía en la preparatoria y recomendado como libro de consulta para los maestros de primaria superior (Meneses, 1998: 622, 658, 711). ${ }^{12}$ También fue autor de un tratado conocido con el título El cultivo del maíz (1937). Schulz fue reconocido por sus contemporáneos como un gran orador; por ejemplo, en 1898 formó parte, junto con Ezequiel A. Chávez y Pablo Macedo, del homenaje al doctor Gabino Barreda con una serie de discursos y poesías (Chávez et al., 1898). Además, recibió diversas distinciones de la Sociedad Mexicana de Geografía y Estadística, la Academia de la Lengua y la Sociedad Científica Antonio Alzate. ${ }^{13}$

A lo largo de su vida, ocupó varios cargos en la administración educativa, incluyendo la dirección de la Escuela Normal para Profesores de Primaria. En 1887 fue encargado del des-

${ }^{6}$ ACHSEP, Secretaría de Estado y del Despacho de Justicia e Instrucción Pública. Expediente personal (Miguel E. Schulz), exp. S2/12, fs. 2-4, 17-19, 21, 39

7 "Obituario..." El Universal, 4 de diciembre de 1922.

${ }^{8}$ ACHSEP, Secretaría de Estado y del Despacho de Justicia e Instrucción Pública, Expediente personal (Miguel E. Schulz), exp. S2/12, f. 83.

9 "Obituario..." El Universal, 4 de diciembre de 1922.

10 "Obituario..." El Universal, 4 de diciembre de 1922.

${ }^{11}$ Este texto fue premiado en la Exposición Universal Colombina de Chicago, 1893, y planteó una concepción innovadora de la Geografía según el Dr. Luis Ignacio Hernández Iriberri, Comentario publicado en su blog, <http://espacio-geografico.over-blog.es/article-comentario-a-apuntes-para-el-curso-de-geografia-primera-edicion-1893-segunda-edicion-1896-de-miguel-enrique-schulz-articulo-2010-555>, (consulta: 25/09/2015).

12 "Obituario..." El Universal, 4 de diciembre de 1922.

13 "Obituario..." El Universal, 4 de diciembre de 1922. 
pacho de la Secretaría de Justicia e Instrucción Pública ${ }^{14}$ y durante muchos años fue vocal de la antigua Junta Directiva de Instrucción Pública, desaparecida en 1902 (Bazant, 1993 35). Para 1904 fue nombrado director interino de la Escuela Nacional Preparatoria, cargo que cubrió por seis meses en ausencia de Manuel Flores. ${ }^{15}$ Según su obituario, "desempeñó con general aprobación el importante puesto de Director de la Escuela Nacional Preparatoria planteando en ella reglas de orden y equidad que merecieron elogios". ${ }^{16}$ Entre otras funciones, estuvo al frente de las comisiones técnicas relativas a la instrucción y educación pública en varios estados y gobiernos de diversas entidades federativas.

Durante la gestión de Justo Sierra en pro de la educación nacional, Miguel E. Schulz formó parte del Consejo Superior de Educación Pública, cuerpo consultivo constituido por hombres eminentes de la vida cultural en México (Meneses, 1998: 601).17 También prestó sus servicios, durante $1904^{18}$ y $1905^{19}$, como oficial primero de la Sección de Instrucción Secundaria, Preparatoria y Profesional de la Secretaría de Estado y del despacho de Justicia y de Instrucción Pública. Cuando se fundó la Secretaría de Instrucción Pública y Bellas Artes (SIPBA), en mayo de 1905 -con Justo Sierra como primer Secretario del ramo-, Schulz tomó protesta por su nombramiento como jefe de la Sección de Estadística, Información y Archivo en dicha secretaría. ${ }^{20}$ Ocupaba este mismo cargo en 1909, cuando publicó el documento de estadística escolar que presentamos en este trabajo (véase apéndice). ${ }^{21}$

En 1910 se fundó la Universidad Nacional de México, en la cual se integraron las escuelas profesionales, la Escuela de Altos Estudios y la Escuela Nacional Preparatoria. Miguel E. Schulz se incorporó a la recién fundada universidad y ocupó varios puestos. Entre fines de 1916 y comienzos de 1917, desempeñó el cargo de rector interino mientras José Natividad Macías, el rector en turno, asistía al Congreso Constituyente (1916-1917). ${ }^{22}$ Schulz formaba parte del grupo de académicos sobresalientes de la Universidad, entre los que se incluían a Ezequiel Chávez, Antonio Caso, Vicente Lombardo Toledano y Manuel Gómez Morín. Todos continuaron ejerciendo su labor como profesores durante el régimen de Francisco I. Madero y aún después de la Decena Trágica, cuando Huerta tomó el poder. A mediados de 1914, Venustiano

${ }^{14}$ ACHSEP, Secretaría de Estado y del Despacho de Justicia e Instrucción Pública. Expediente personal (Miguel E. Schulz), $\exp . \mathrm{S} 2 / 12$, fs. 11 .

${ }^{15}$ ACHSEP, Secretaría de Estado y del Despacho de Justicia e Instrucción Pública. Expediente personal (Miguel E. Schulz), exp. S2/12, fs. 42-44.

16 "Obituario..." El Universal, 4 de diciembre de 1922.

${ }^{17}$ Boletín de Instrucción Pública, t. IV, núm. 5, 1905: 755 y t. XI, num. 2, 1908: 410.

${ }^{18}$ ACHSEP, Secretaría de Estado y del Despacho de Justicia e Instrucción Pública. Expediente personal (Miguel E. Schulz), exp. S2/12, fs. 35-38, 49-50.

\footnotetext{
${ }^{19}$ AGN, Instrucción Pública y Bellas Artes, c. 250, exp. 7, f. 1, 1905.

${ }^{20}$ AGN, Instrucción Pública y Bellas Artes, c. 250, exp. 7, f. 66, 1905.

${ }^{21}$ Archivo Histórico del Estado de Tlaxcala, FRRO, Instrucción Pública, c. 301, exp. 25

${ }^{22}$ Compendio de Legislación Universitaria, pp. 69-70. <http://biblio.juridicas.unam.mx/libros/1/254/25.pdf>
} 
Carranza ocupó la Ciudad de México y nombró a Félix Palavicini como Secretario de Instrucción Pública y Bellas Artes; se reemplazaron entonces las autoridades universitarias y fueron despedidos muchos profesores de la preparatoria por haber colaborado con Huerta o favorecido a Villa. Según Garciadiego, Schulz, a pesar de ser considerado "apolítico", fue uno de los que presentó su renuncia en la Escuela Nacional Preparatoria, tal vez voluntariamente, debido a "la actitud antiintelectualista del carrancismo" (Garciadiego, 1996: 275).

En 1917, la nueva Constitución Política abolió la Secretaría de Instrucción Pública y Bellas Artes, y la Universidad quedó bajo una dirección administrativa y perdió la Escuela Nacional Preparatoria, además de varios institutos. En este contexto, Miguel Schulz, habiendo sido director de la Escuela de Altos Estudios de la Universidad, creó junto con otros universitarios una nueva preparatoria "libre", donde los profesores impartían clases a unos 500 estudiantes (Garciadiego, 1996: 381-383; 355-359). Esta institución se cerró en 1920, cuando el régimen del sonorense restableció la Secretaría de Educación Pública y le devolvió a la Universidad la rectoría sobre la Escuela Nacional Preparatoria. Miguel E. Schulz falleció dos años después.

Los documentos que publicamos fueron impresos en 1909 por la Secretaría de Instrucción Pública y Bellas Artes y constituyen fuentes invaluables para conocer las condiciones de la educación formal, durante el periodo comprendido entre la Restauración de la República y el inicio de la Revolución (véase Apéndice). Schulz fue capaz de sistematizar las estadísticas de José Díaz Covarrubias (1993 [1875])-ministro de Justicia e Instrucción Pública en el gabinete de Lerdo de Tejada-, que reportan el número de escuelas en 1875 (Latapí 1993: 129), y las que él mismo recogió de los estados en 1907. Lo interesante de estos cuadros es que permiten comparar la cantidad de escuelas por kilómetro cuadrado y por población en cada entidad, además de proporcionar datos en los que se observa el crecimiento de la oferta escolar de cada estado durante el Porfiriato. Contienen, así, algunas sorpresas en cuanto a las posiciones relativas en la escolarización entre los estados durante aquel periodo; por ejemplo, Guerrero, que ocupaba una posición relativamente alta para ese tiempo.

Es importante considerar tanto las posibilidades como las limitaciones en el uso de estos datos, como base para establecer comparaciones con información correspondiente a años posteriores. Sería muy interesante calcular nuevamente el número de escuelas por área y por población en cada estado, para conocer los avances absolutos y relativos de la oferta escolar a lo largo del siglo xx. Sin embargo, es necesario recordar que esta institución cambió su configuración durante dicho periodo. Resulta complicado comparar los datos referentes a escuelas de mujeres y varones por separado -que son las que predominaban entre 1875 y 1907-, con aquellos posteriores a la Revolución, en su mayoría relativos a escuelas mixtas, pues al agruparse ambos sexos aparece un descenso en el número total de los planteles. Esto ocurre también con la desaparición de las pequeñas escuelas rurales en algunas regiones, lo que ocasionó que los estudiantes se concentraran en escuelas más grandes en 
los pueblos, pues a finales del siglo xix la mayoría de los centros de enseñanza eran unitarios, es decir, unidocentes. Poco a poco se incrementó el número de maestros, sobre todo en las escuelas graduadas en las urbes (Rockwell y Garay, 2014).

Las estadísticas centradas en la población escolar y el número de escuelas omiten datos tan importantes como el criterio empleado para definir la edad escolar, la matrícula real y el número de grados y maestros promedio por escuela. Incluso habría que tomar en cuenta qué unidades se consideraron como "escuelas" en cada censo o informe: ¿se incluyeron en los datos las escuelas particulares, municipales, estatales y federales, anexas o económicas, de turno matutino o vespertino? No es tan fácil saberlo. Por todo lo anterior, hay cierto riesgo en comparar las estadísticas escolares entre dos periodos históricos diferentes.

Con todas estas precauciones, esperamos que el cuadro sea de utilidad para los historiadores que intenten reconstruir los avances -y los retrocesos, pues en unos casos disminuyó el número de escuelas por población total- de la cobertura escolar en México durante los últimos dos siglos.

\section{Fuentes}

Archivos

ACHSEP, Secretaría de Estado y del Despacho de Justicia e Instrucción Pública, Expediente personal (Miguel E. Schulz), exp. S2/12. 122 fs. 1887-1917. Nombramientos, ceses y otros documentos relativos de Miguel E. Schulz durante los años de servicio en la Escuela Nacional Preparatoria y la Escuela Normal de Profesores.

AGN, Instrucción Pública y Bellas Artes, Caja 316, exp. 8, 6 fs. 1870. Distrito Federal. Academia de San Carlos. Pensiones a Manuel Ocarraza, Rodrigo Gutiérrez, Félix Parra, José María Ortega, Jacinto Enciso, Miguel Schulz y Agustín Ocampo, como premios. Ramo Instrucción Pública y Bellas Artes, Caja 250, exp. 7, 7 fs. 1905. Distrito Federal, Sección de Instrucción Preparatoria y Profesional. Nombramiento a Jesús Acevedo en lugar de Miguel E. Schulz, como oficial primero de la Sección.

Ramo Instrucción Pública y Bellas Artes, Caja 296, exp. 12, 14 fs. 1919. Distrito Federal, Universidad Nacional de México. Comisión a Ángel Grosso, Antonio Caso, Miguel E. Schulz y Rogación Ortega, para organizar acto conmemorativo hacia Jesús Díaz de León.

Ramo Instrucción Pública y Bellas Artes, Caja 296, exp. 13, 1 f. 1919. Distrito Federal, Universidad Nacional de México. Comisión a Miguel E. Schultz, José León Martínez y Francisco Lasci, para dictaminar sobre los pagos e inscripciones de alumnado universitario.

AHET, FRRO, Instrucción Pública, c. 301, exp. 25. 1909, Cuadro en que se resume el estado en que en 1874 se encontraba la Instrucción Pública.

Hemerografía

Boletín de Instrucción Pública. Órgano de la Secretaría del Ramo (1905), t. IV, núm. 5.

Boletín de Instrucción Pública. Órgano de la Secretaría del Ramo (1908), t. XI, núm. 2.

"Obituario de Miguel E. Schulz", El Universal, 4 de diciembre de 1922, p. 2. 
Bibliografía

Bazant, Mílada (1993), Historia de la educación durante el porfiriato, México, El Colegio de México/ Centro de Estudios Históricos.

Díaz Covarrubias, José (1993) [1875], La Instrucción Pública en México. Estado que guardan la instrucción primaria, la secundaria y la profesional en la República. Progresos realizados, mejoras que deben introducirse, edición facsimilar, México, Conacyt-Miguel Ángel Porrúa.

Chávez, Ezequiel A., José Ramos, Miguel E. Shultz y Pablo Macedo (1898), Discursos y poesía en honor del Dr. Don Gabino Barreda. Marzo 10 de 1898, México, Tipografía T. Conzález.

Garciadiego Dantan, Javier (1996), Rudos contra científicos. La Universidad Nacional durante la Revolución mexicana, México, El Colegio de México/Centro de Estudios Históricos-UnAm/Centro de Estudios sobre la Universidad.

Latapí, Pablo (1993), "J. Díaz Covarrubias, 'La Instrucción Pública en México. Estado que guardan la instrucción primaria, la secundaria y la profesional en la República. Progresos realizados, mejoras que deben introducirse'", Reseña, Revista Latinoamericana de Estudios Educativo, vol. XXIII, núm.l, pp. 129-133.

Meneses Morales, Ernesto (1998), Tendencias educativas oficiales en México. 1821-1911. La problemática de la educación mexicana en el siglo xIx y principios del siglo xx, México, Universidad Iberoamericana/ Centro de Estudios Educativos.

Rockwell, Elsie y Claudia Garay Molina (2014), "Las escuelas unitarias en México en perspectiva histórica: un reto aún vigente", Revista Mexicana de Historia de la Educación, vol. II, núm. 3, pp. 1-24.

Schulz, Miguel E. (1937), El cultivo del maíz, México, Editorial de Izquierda de la Cámara de Diputados. (1896) [1892], Apuntes para el Curso de Geografía, México, José Joaquín Terrazas e Hijas. (1895), Curso de Geografía General, México, Librería de la viuda de Ch. Bouret.

Referencias electrónicas

"Don Miguel E. Schulz, Rector de la Universidad Nacional Autónoma de México (UNAM)", disponible en: <https://es.wikipedia.org/wiki/Miguel_E._Schulz\#mediaviewer/File:Miguel_E_Schultz.jpg>, actualización: febrero de 2015; (consulta: 25/09/2015).

"Don Miguel E. Schultz, (1851-1922) Interino del 23 de noviembre de 1916 al 3 de mayo de 1917", disponible en: <http://biblio.juridicas.unam.mx/libros/1/254/25.pdf> (consulta: 13/02/2015). 


\section{Apéndice}

\section{Cuadros que resumen el estado de la instrucción}

pública en 1874 y 1907, por estado de la República

Mexicana. ${ }^{1}$

\section{Miguel E. Schulz}

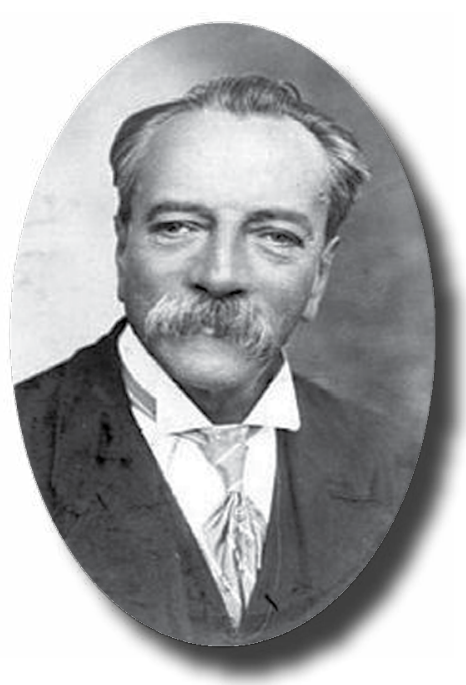

${ }^{1}$ Archivo Histórico del Estado de Tlaxcala (en adelante AHET), Fondo Revolución y Régimen Obregonista, Sección Instrucción Pública (en adelante FRRO/IP), c. 301, exp. 75. 1909. Agradecemos a la etnohistoriadora Lourdes Solares Ramos por la ubicación del ejemplar original del cuadro en el Archivo Histórico del Estado de Tlaxcala y la toma de fotografías. A la antropóloga Yolanda Ramos Galicia, directora del Colegio de Historia y a la historiadora Liliana Zamora Poire, Jefa del Departamento del Archivo Histórico del Estado de Tlaxcala, por facilitar la versión escaneada de los cuadros y el permiso para publicarlos en la Revista Mexicana de Historia de la Educación. Agradecemos a la Mtra. Verónica Arellano por proporcionarnos el "Obituario de Miguel E. Schulz", El Universal, 4 de diciembre de 1922, pp. 1-2. 



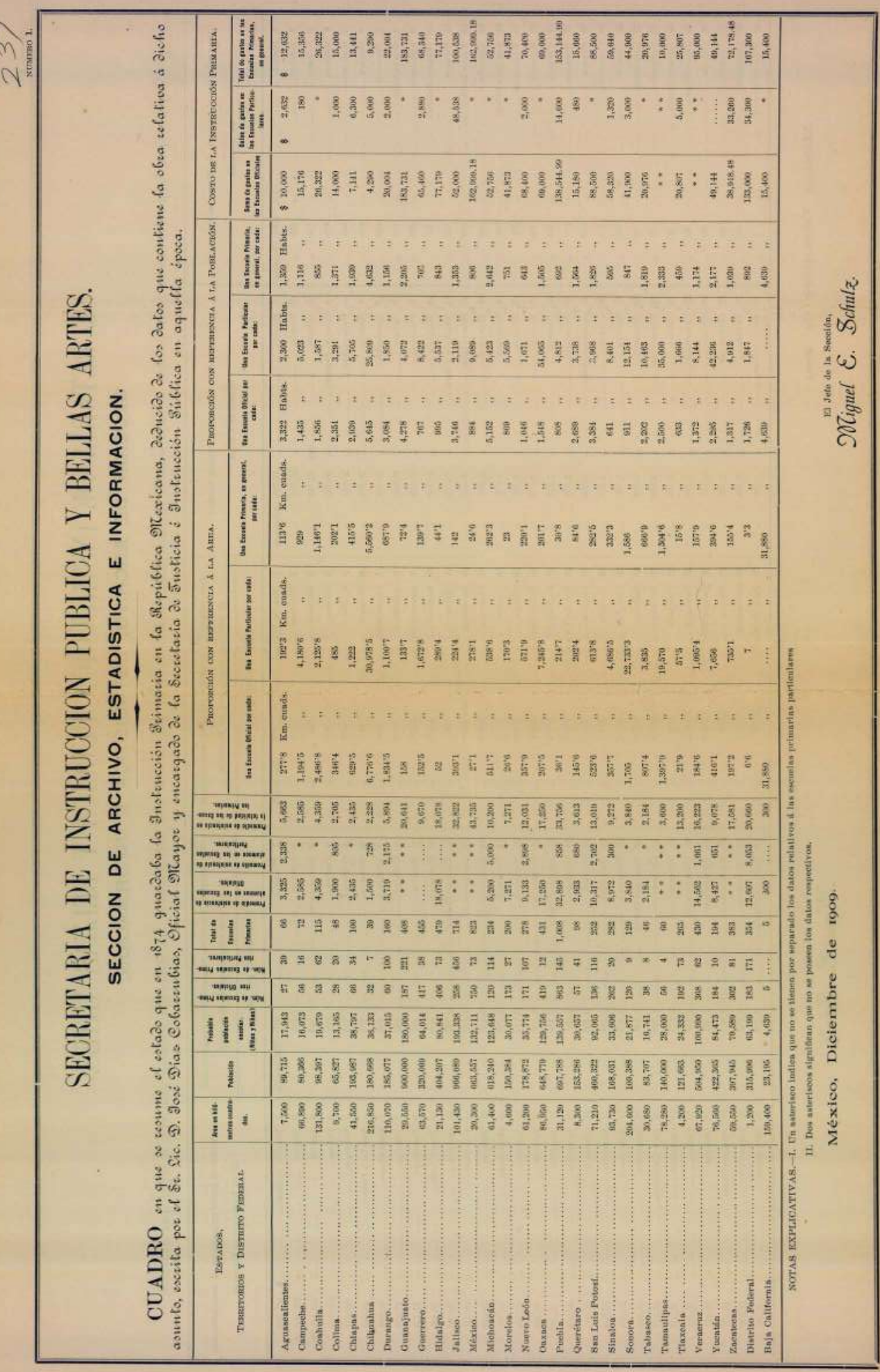




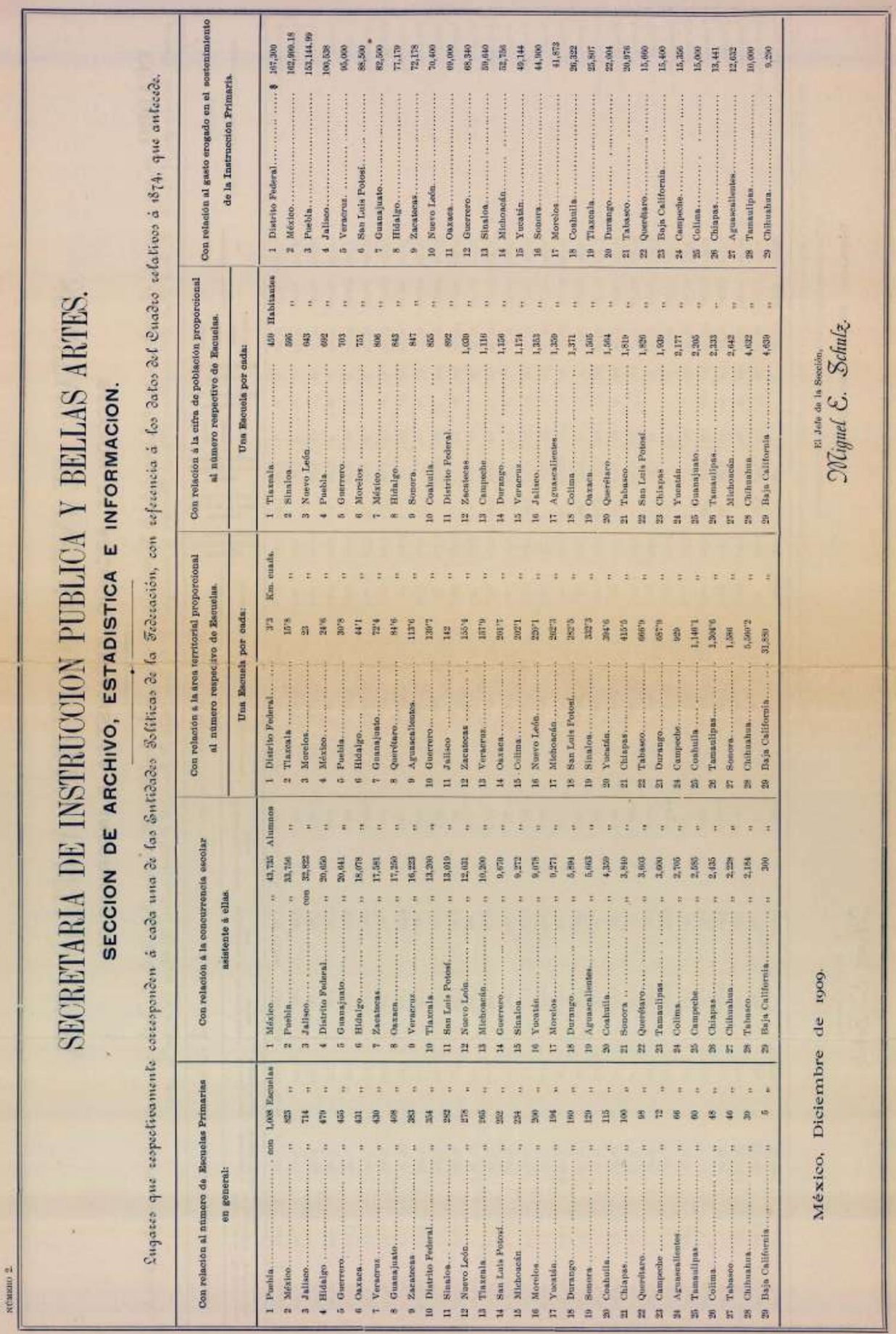




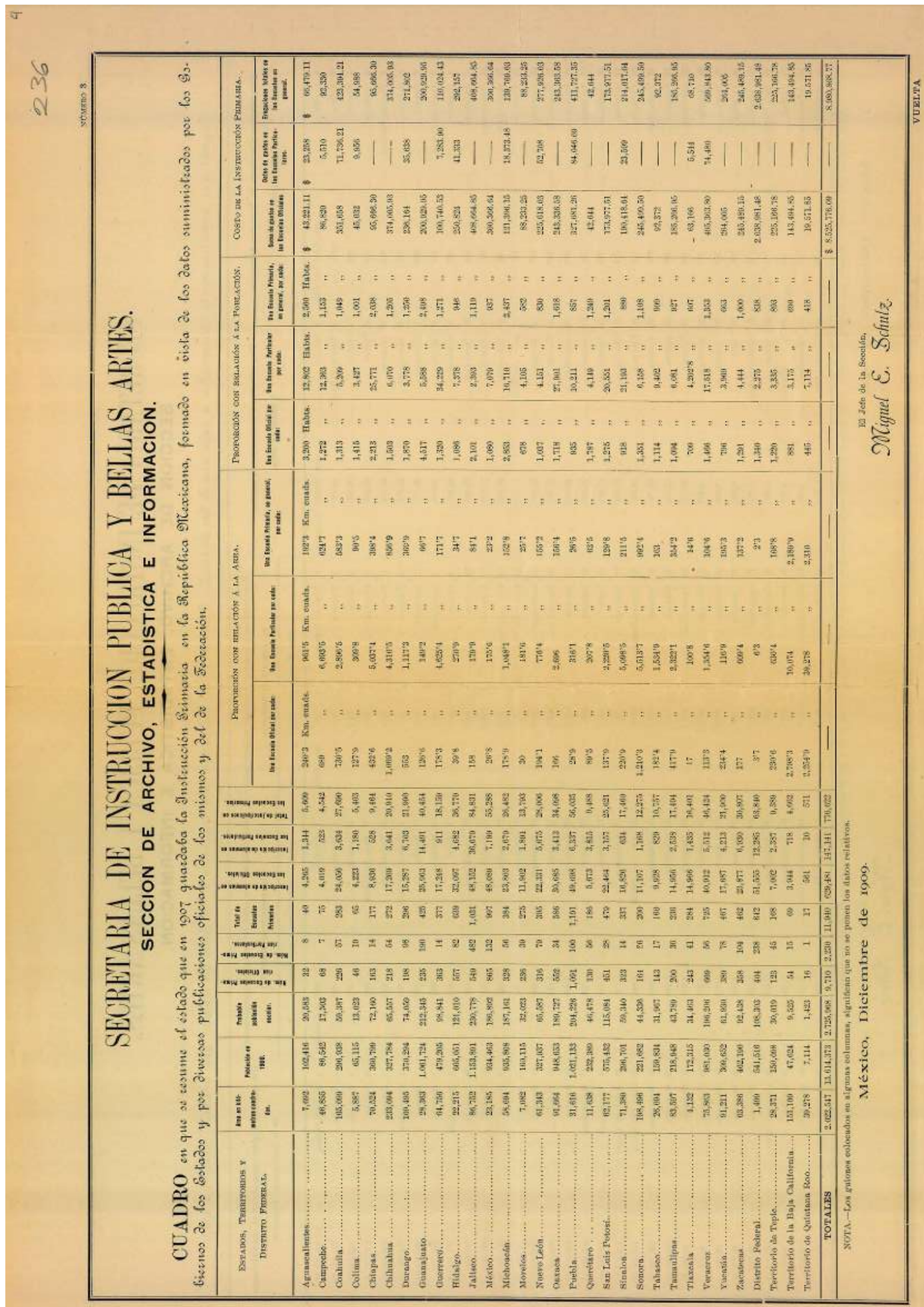




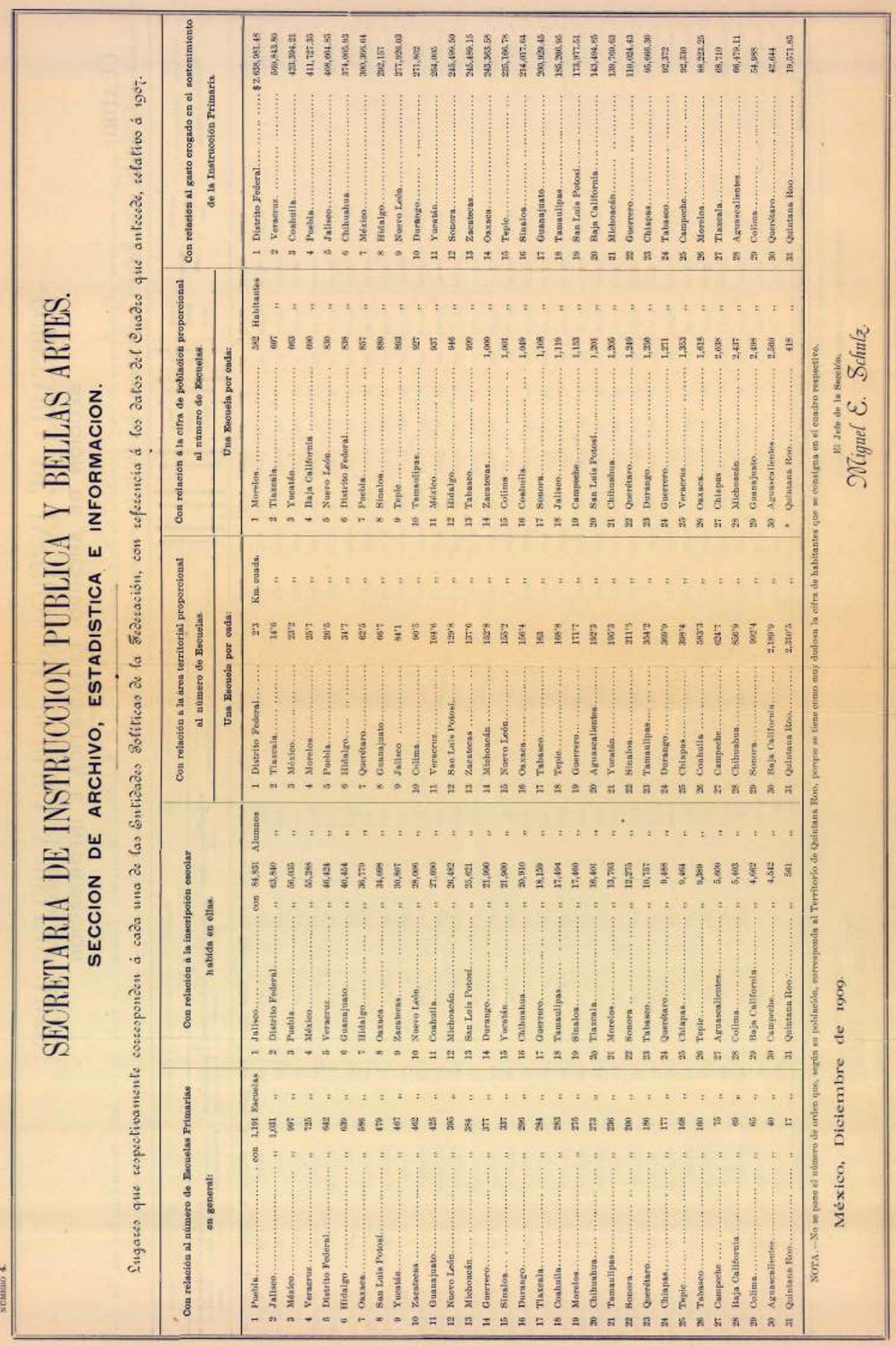

